Journal for ImmunoTherapy of Cancer

To cite: Wang H, Ma H, Sové RJ, et al. Quantitative systems pharmacology model predictions for efficacy of atezolizumab and nab-paclitaxel in triplenegative breast cancer. Journal for ImmunoTherapy of Cancer 2021;9:e002100. doi:10.1136/ jitc-2020-002100

Accepted 03 January 2021

\title{
Quantitative systems pharmacology model predictions for efficacy of atezolizumab and nab-paclitaxel in triple-negative breast cancer
}

\author{
Hanwen Wang (D) , ${ }^{1}$ Huilin Ma (D) , ${ }^{1}$ Richard J Sové, ${ }^{1}$ Leisha A Emens, ${ }^{2}$ \\ Aleksander S Popel (iD ${ }^{1,3}$
}

\section{ABSTRACT}

Background Immune checkpoint blockade therapy has clearly shown clinical activity in patients with triplenegative breast cancer, but less than half of the patients benefit from the treatments. While a number of ongoing clinical trials are investigating different combinations of checkpoint inhibitors and chemotherapeutic agents, predictive biomarkers that identify patients most likely to benefit remains one of the major challenges. Here we present a modular quantitative systems pharmacology (QSP) platform for immuno-oncology that incorporates detailed mechanisms of immune-cancer cell interactions to make efficacy predictions and identify predictive biomarkers for treatments using atezolizumab and nabpaclitaxel.

Methods A QSP model was developed based on published data of triple-negative breast cancer. With the model, we generated a virtual patient cohort to conduct in silico virtual clinical trials and make retrospective analyses of the pivotal IMpassion130 trial that led to the accelerated approval of atezolizumab and nab-paclitaxel for patients with programmed death-ligand 1 (PD-L1) positive triplenegative breast cancer. Available data from clinical trials were used for model calibration and validation.

Results With the calibrated virtual patient cohort based on clinical data from the placebo comparator arm of the IMpassion130 trial, we made efficacy predictions and identified potential predictive biomarkers for the experimental arm of the trial using the proposed QSP model. The model predictions are consistent with clinically reported efficacy endpoints and correlated immune biomarkers. We further performed a series of virtual clinical trials to compare different doses and schedules of the two drugs for simulated therapeutic optimization. Conclusions This study provides a QSP platform, which can be used to generate virtual patient cohorts and conduct virtual clinical trials. Our findings demonstrate its potential for making efficacy predictions for immunotherapies and chemotherapies, identifying predictive biomarkers, and guiding future clinical trial designs.

\section{BACKGROUND}

Triple-negative breast cancer (TNBC) is an aggressive subtype of breast cancer that lacks expression of estrogen receptor, progesterone receptor, and human epidermal growth factor receptor-2 (HER-2). ${ }^{1}$ In the past decade, immune checkpoint blockade therapies have shown promising efficacy in patients with melanoma and non-small cell lung cancer, but there have been no approved immunotherapy strategies for patients with TNBC until recently. ${ }^{2}$ In 2019, the combination of a monoclonal anti-PD-L1 antibody, atezolizumab, and a chemotherapeutic agent, nanoparticle albumin-bound (nab)paclitaxel, received accelerated approval from the US Food and Drug Administration as first-line treatment for patients with unresectable locally advanced or metastatic TNBC that is PD-L1 immune cell positive, based on results from the IMpassion130 trial (NCT02425891). ${ }^{34}$ Whereas response rates for patients with advanced TNBC ranged from $0 \%$ to $24 \%$ with single-agent atezolizumab, the IMpassion130 trial reported a response rate of $55 \%$ with the combination of atezolizumab and nab-paclitaxel. ${ }^{5}$ Importantly, the trial showed clinically meaningful improvements in median progression-free and overall survival in PD-L1 immune cell (IC)-positive patients with the addition of atezolizumab to nab-paclitaxel, identifying PD-L1 expression on tumor-infiltrating ICs as a clinically relevant biomarker associated with benefit from this combination regimen. ${ }^{34}$ While the results of IMpassion130 established immunotherapy as a treatment option for some patients with advanced $\mathrm{TNBC}$, it is critical to investigate additional cytotoxic agents to be combined with atezolizumab and other immunotherapies, and further evaluate biomarkers in both PD-L1-positive and PD-L1-negative patient cohorts. $^{6}$

Nab-paclitaxel is a taxane-based chemotherapeutic agent, which is commonly used 
as a first-line treatment for patients with metastatic breast cancer. ${ }^{7}$ The addition of albumin facilitates the transport of nab-paclitaxel into the tumor interstitium through albondin (gp60), an albumin receptor, on endothelial cells. Furthermore, the overexpression of osteonectin (SPARC) on TNBC cells, which has a high affinity with albumin, enhances the uptake of nab-paclitaxel and the release of paclitaxel near the cancer cells. ${ }^{89}$ These mechanisms are thought to contribute to the higher intratumoral concentration of nab-paclitaxel compared with paclitaxel and its other derivatives in TNBC, which was confirmed by population pharmacokinetic (PK) models and preclinical studies. ${ }^{10}{ }^{11}$ Various pharmacodynamic (PD) effects of nab-paclitaxel on TNBC have been observed in preclinical studies, including its cytotoxicity towards cancer and endothelial cells, and upregulation of vascular endothelial growth factor A (VEGF-A) expression, which provides a rationale for combination therapies with anti-VEGF agents such as bevacizumab. ${ }^{12-15}$ In IMpassion130, a dose of $100 \mathrm{mg} / \mathrm{m}^{2}$ nab-paclitaxel was administered days 1,8 and 15 every 28 days along with 840 mg atezolizumab or placebo days 1 and 15 to 902 patients with advanced TNBC. ${ }^{34}$ This combination was supported by the following rationale: the cytotoxic activity of nabpaclitaxel enhances tumor-specific antigen release, with and uptake by and subsequent maturation of antigenpresenting cells (APCs) in the tumor and tumor-draining lymph nodes (TDLNs), and atezolizumab reverses $\mathrm{T}$ cell suppression by selectively blocking interactions between PD-L1 and programmed death 1 (PD-1) on ICs and cancer cells, as PD-L1 can be expressed in TNBC. ${ }^{16}$

To accompany clinical efforts to develop therapies and informative biomarkers that improve clinical outcomes, multiple quantitative systems pharmacology (QSP) models have been developed using various approaches. ${ }^{17-19}$ Hardiansyah et $a l^{19}$ proposed a model to simulate cellular kinetics and cytokine profiles in patients with chronic lymphocytic leukemia receiving chimeric antigen receptor $\mathrm{T}$ cell therapy, which was trained by patient-level data. Betts et $a l^{18}$ presented a model to predict the efficacy of CD3-bispecific antibody therapy using in silico, in vivo and in vitro data across species. Gong $e a^{17}$ built a spatial model to simulate interactions between cancer cells and stromal cells and predict the efficacy and biomarkers of immunotherapy based on digital pathology data. In our previous studies, we developed an ordinary differential equation-based QSP platform to predict results of a clinical trial in HER-2 negative breast cancer using anti-PD-1 and anti-CTLA- 4 antibodies with an epigenetic modulator, using in vitro and in vivo data from preclinical studies. ${ }^{20}$ By expanding our previous model, ${ }^{20}$ we conducted a virtual clinical trial of nabpaclitaxel and atezolizumab and compared our simulated results with published population-level data from IMpassion130. The virtual clinical trial aims to generate a virtual patient cohort that can be mapped to a clinical cohort in a specific trial. While there exist various methodologies in the virtual patient generation, the optimization of these algorithms is under active investigation..$^{21-25}$ In this study, we aim to determine the relationship between our QSP platform and the virtual cohort with the patient cohort and results of the clinical trial. We discuss the limitations related to our choice of methodology, which need to be taken into account while interpreting the present numerical results and comparisons.

\section{METHODS}

\section{Model overview}

The present model is adapted from our previously published QSP platform ${ }^{20}$ using the SimBiology toolbox in MATLAB (MathWorks, Natick, Massachusetts, USA), which comprises four compartments: central, peripheral, tumor, and TDLNs. As a modular model, it was introduced with eight modules that describe the kinetics and dynamics of effector $\mathrm{T}$ cells (Teff), regulatory $\mathrm{T}$ cells (Treg), cancer cells, APCs, tumor-specific neoantigens and tumor-associated self-antigens, immune checkpoints, myeloid-derived suppressor cells (MDSCs), and therapeutic agents. ${ }^{20}$ In this study, we added a chemotherapeutic module for nab-paclitaxel, modified tumor growth and PD-L1 dynamics, and further expanded the $\mathrm{T}$ cell module for naive $\mathrm{T}$ cell kinetics in this study. The dynamics of the major species in the model are illustrated in figure 1A, including: immune activation of naive $\mathrm{CD}^{+}$and $\mathrm{CD} 4^{+} \mathrm{T}$ cells in the TDLNs by tumor-specific neoantigens and tumor-associated self-antigens; $\mathrm{T}$ cell trafficking throughout the body; immune evasion mediated by immune checkpoints, Treg, MDSCs, and antiinflammatory cytokines; and PK/PD of drugs of interest. The modules were written using MATLAB scripts and incorporated 271 parameters, 140 ODEs, and 54 algebraic equations in total. Details of all the modules are elaborated in the online supplemental methods, and full lists of model parameters, reactions, algebraic equations, and cellular and molecular species are presented in the online supplemental tables S1-S6).

\section{Model initiation and simulation settings}

The model workflow is illustrated in figure $1 \mathrm{~B}$ with five steps: (1) load baseline parameters into the MATLAB workspace and initialize the SimBiology model with initial compartments and simulation settings; (2) add modules that include model species, rules, and events by calling the corresponding MATLAB functions; (3) simulate to find the initial condition with baseline parameters and a desired initial tumor diameter; (4) create SimBiology dose objects for drugs of interest and simulate therapy; and (5) generate a number of parameter sets to perform virtual clinical trials and subsequent analyses. The baseline parameters are estimated or fitted based on in vitro and in vivo experimental data generated using TNBC cell lines from literature (online supplemental methods). After model initialization with baseline parameters and required modules, a simulation is performed with a small number of cancer cells and a desired initial tumor 
A.

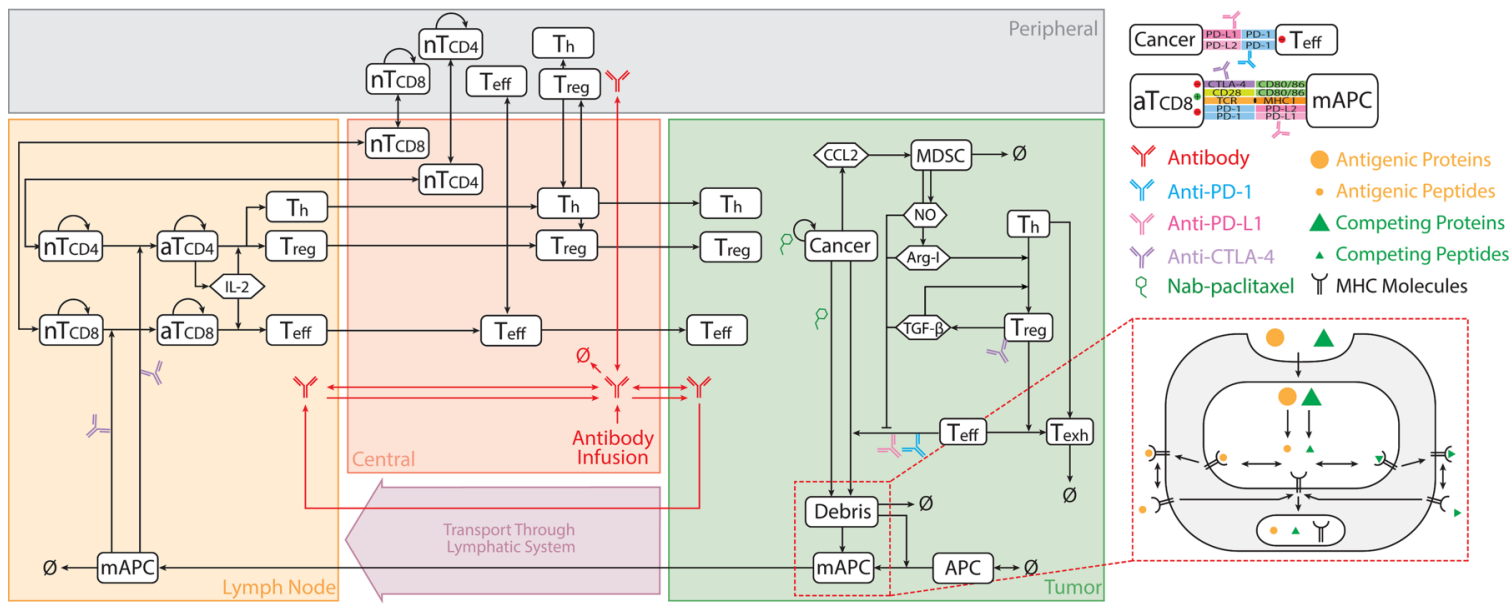

B.

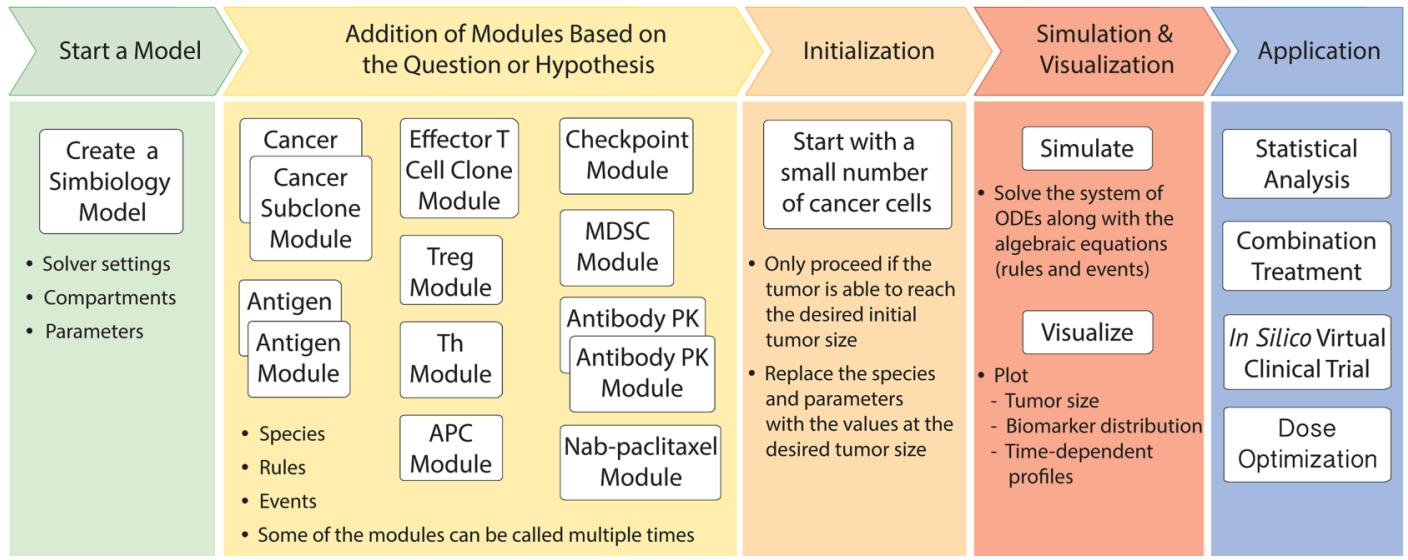

Figure 1 QSP model diagram (A) and workflow (B). The model is composed of four compartments: central, peripheral, tumor, and tumor-draining lymph node, which describe cycles of immune activation in lymph nodes, $T$ cell trafficking to the tumor, killing of cancer cells, immune evasion, and antigen release and lymphatic transport. ARG-I, arginase I; AT, activated T cell; MAPC, mature antigen presenting cell; NO, nitric oxide; QSP, quantitative systems pharmacology; NT, naïve T cell; TEFF, effector T cell; TH, T helper cell; Treg, regulatory T cell. Modified from ref 47.

diameter. The desired initial tumor diameter corresponds to the pretreatment tumor size of a patient in the clinical trial. Once the initial tumor diameter is reached, the amount of model species and parameter values are saved as the initial condition, which corresponds to the pretreatment condition of the patient in the clinical trial. The model is then reinitialized with the previously saved initial condition and simulated with drugs of interest. A sample of model outputs is presented in online supplemental figure S1.

\section{Virtual patient generation and virtual clinical trial}

The virtual clinical trial, or in silico clinical trial, aims to predict the efficacy of drugs of interest in patients with particular conditions (eg, melanoma and TNBC) using the virtual patient cohort. ${ }^{226}$ To generate a virtual patient cohort that resembles the clinical population, a subset of model parameters is selected to be varied, while others are kept at the baseline level. The selected parameters, such as antigen binding affinity, the number of tumorspecific T cell clones, initial tumor diameter, and $\mathrm{PK} / \mathrm{PD}$ parameters of nab-paclitaxel aim to capture the interindividual variabilities in real patients. The distributions of these parameters are estimated based on available literature data on TNBC. With the majority of the model parameter values estimated based on in vitro and in vivo data, we use data from clinical trials to further calibrate the distribution of varied model parameters. Specifically, the published clinical results from NCT01375842 and the placebo comparator arm of the IMpassion130 trial are used for model calibration, and the results from the experimental arm of the IMpassion130 trial are used for model validation.

To perform a virtual clinical trial, the model is first initialized as described by steps 1-2. The values of selected parameters are randomly generated based on the calibrated parameter distributions using Latin Hypercube Sampling (LHS), with each parameter set representing a potential virtual patient. Each randomly assigned parameter set is then plugged into the model to simulate tumor response to the therapy following steps 3-4. For postprocessing after the simulation, the potential virtual patients are filtered by the acceptance criteria based on their pretreatment $\mathrm{T}$ cell densities so that their values fall into the physiologically plausible range in patients with 
TNBC, ${ }^{21}$ which are reported by clinical measurements. ${ }^{27} 28$ For comparison purpose, the two-dimensional (2-D) $\mathrm{T}$ cell density reported by the clinical measurements are converted to three-dimensional (3-D) density using the equation from Mi et al..$^{29}$ In this study, the total number of 900 virtual patients are generated on calibration for efficacy predictions and statistical analyses. The study serves as the first retrospective analysis of the IMpassion 130 trial using virtual patient cohorts. ${ }^{4}$ To match the clinical settings, the simulation time is set to be 400 days, which corresponds approximately to the median follow-up time of 12.9 months in the IMpassion130 trial. ${ }^{4}$ Additionally, the simulated tumor diameters are recorded every 8 weeks, which corresponds to the frequency of tumor measurements in the clinical trial.

\section{Statistical analysis}

Global uncertainty and sensitivity analysis are performed with LHS and Partial Rank Correlation Coefficient methods to examine the impact of varied parameters on model observations. ${ }^{30}$ The objective response rate (ORR) and duration of response (DOR) are predicted based on RECIST V.1.1, ${ }^{31}$ and the 95 percentile bootstrap CIs are calculated for comparison between model predictions and clinical results. In subgroup analysis, 95\% AgrestiCoull CIs are estimated for the ORR predictions based on the normal approximation for the binomial distribution. For comparison of model observations in subgroups of different response status and treatment regimens, the Wilcoxon test is performed using ggpubr package in RStudio V.1.2. ${ }^{32}$

\section{RESULTS}

\section{Efficacy predictions of the PD-L1 inhibitor}

In the previous study, we made prospective predictions of response rates for PD- 1 and CTLA-4 inhibitors, and an epigenetic modulator, using the same dose regimens of an ongoing clinical trial in patients with HER-2 negative breast cancer at the time. ${ }^{20}$ Our predicted response rate of anti-PD-1 monotherapy fell into the range reported by multiple clinical trials of PD-1 inhibitors, such as nivolumab and pembrolizumab, in patients with breast cancer. ${ }^{5}$ To calibrate our modifications made in this study, we first conduct a virtual clinical trial using a PD-L1 inhibitor, atezolizumab, in virtual patients with TNBC and compare with the results from a phase I clinical trial reported by Emens et al. ${ }^{33}$ In this phase I trial, 116 patients with metastatic TNBC were enrolled and received atezolizumab treatment. Among the 116 patients, 93 patients received $15 \mathrm{mg} / \mathrm{kg}$ atezolizumab, 1 patient received 20 $\mathrm{mg} / \mathrm{kg}$ atezolizumab and 22 patients received $1200 \mathrm{mg}$ atezolizumab, every 3 weeks. One hundred fifteen of the 116 patients had evaluable disease and were included to calculate the ORR by RECIST V.1.1. ${ }^{31}$ In summary, 11 patients had partial or complete response, 15 patients had stable disease, 73 patients had progressive disease and 16 patients died within 6 weeks after treatment started. The results correspond to an ORR of $10 \%$, a percentage of stable disease of $13 \%$, and a percentage of progressive disease of $77 \%$.

To perform a virtual clinical trial that is comparable with the phase I trial, we aim to reproduce the clinical settings in our model simulations. Since most of the patients received $15 \mathrm{mg} / \mathrm{kg}$ or $1200 \mathrm{mg}$ atezolizumab, we use 1200 $\mathrm{mg}$ atezolizumab every 3 weeks in the virtual clinical trial due to a lack of information of patients' body weights. On calibration of the parameter distributions in the virtual patient generation, we generate 900 virtual patients based on our filter criteria specified in the Methods. To match the number of patients enrolled in the clinical trial, we perform 1000 bootstrap resampling of 99 virtual patients from the virtual patient cohort and calculate the bootstrap medians and the 95 percentile bootstrap CIs for efficacy predictions. The resulting median ORR is $13.1 \%$ with a CI from $6.1 \%$ to $20.2 \%$; the median percentage of stable disease is $64.7 \%$ with a CI from $52.5 \%$ to $73.7 \%$; and the median percentage of progressive disease is $23.2 \%$ with a CI from $14.1 \%$ to $32.3 \%$. By comparison, the clinically observed ORR from the phase I trial falls into the bootstrap CI reported by the model prediction. However, the model overestimates the percentage of stable disease and underestimates the percentage of progressive disease when compared with clinical observations. This shift of model prediction from progressive disease to stable disease is, at least partially, due to the lack of model prediction for metastasis. As reported by Emens $e t a l,{ }^{33}$ a number of patients are considered to have progressive disease due to newly detected lesions (ie, newly detected metastases), even with a reduction of overall tumor burden.

To better virtualize the difference between the model predictions and the clinical results, we plot the rate of response and the best overall response as the spider plot and waterfall plot, respectively, in figure 2A. The figure demonstrates the capability of virtual clinical trials to capture the interindividual variabilities, as the tumor dynamics of the virtual patient cohort resemble those reported by the clinical trial. To further explore the results, we plot the distributions of potential predictive biomarkers in figure 3 and compare their differences between responders and non-responders. As shown in the figure, the number of tumor-specific $\mathrm{T}$ cell clones, binding affinity $\left(\mathrm{K}_{\mathrm{D}}\right)$ of neoantigens, $\mathrm{CD} 8^{+}$and $\mathrm{CD} 4^{+}$ $\mathrm{T}$ cell densities, $\mathrm{CD} 8^{+} /$Treg and $\mathrm{CD} 4^{+} /$Treg ratios are significantly higher in responders, which shows consistency with our previous model predictions and clinical evidence. ${ }^{62034-36}$

\section{Virtual clinical trial of atezolizumab and nab-paclitaxel}

To specifically calibrate the parameters related to chemotherapy, we performed another virtual clinical trial using the treatment regimens from the IMpassion130 trial. The trial contains two treatment arms: placebo plus nabpaclitaxel (placebo comparator) arm and the atezolizumab plus nab-paclitaxel (experimental) arm. In the 
A.

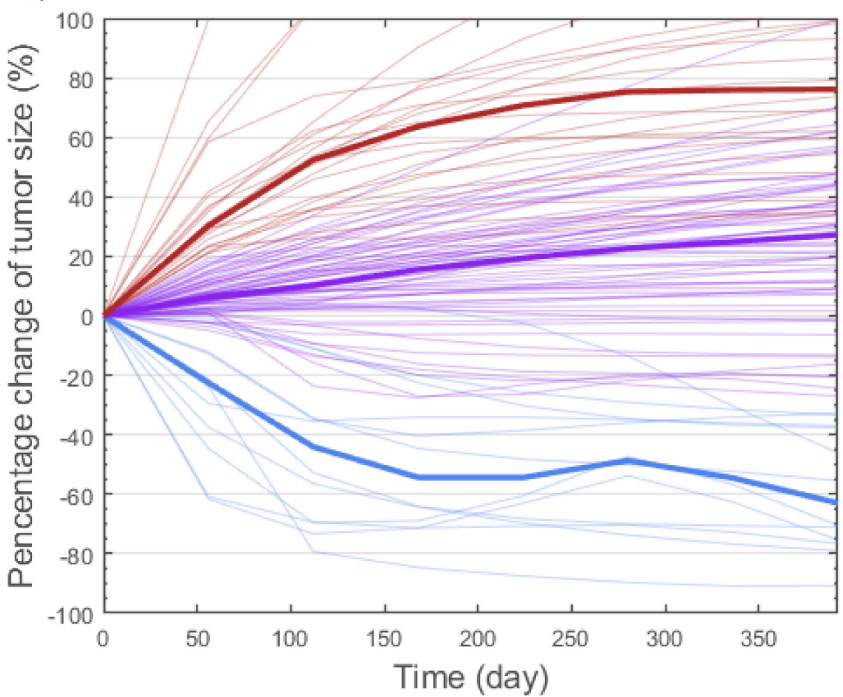

B.

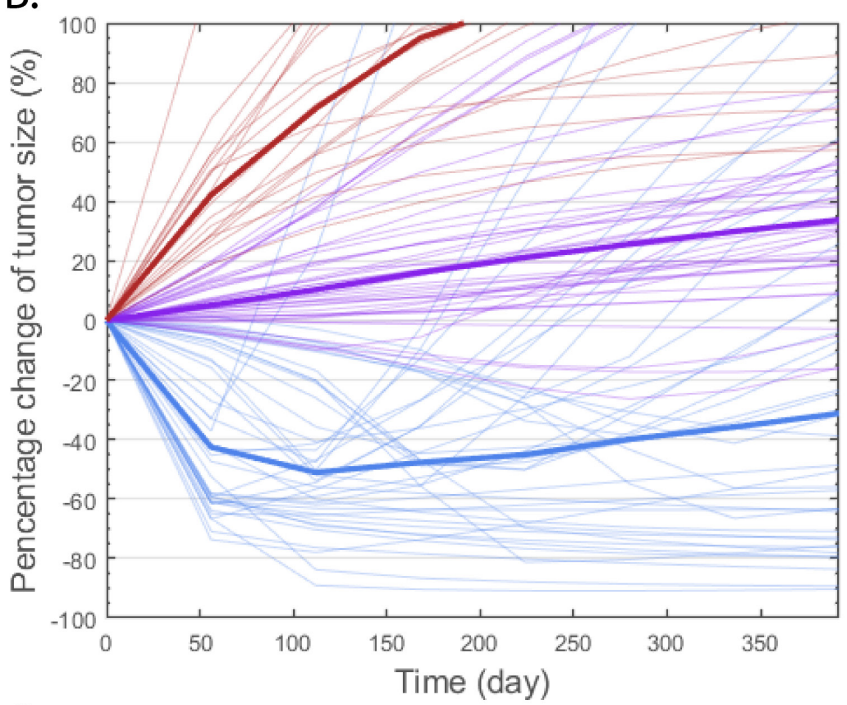

C.

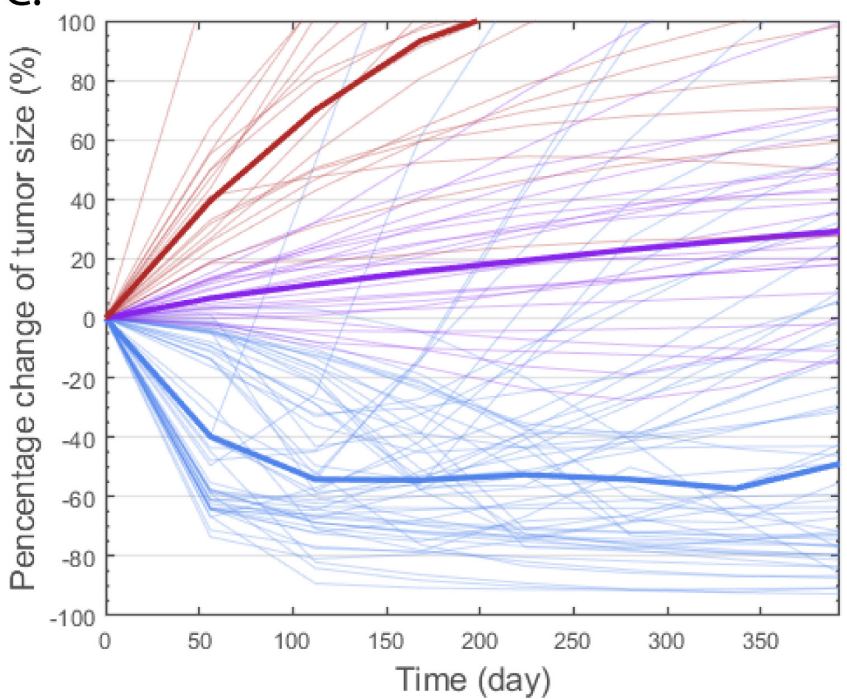

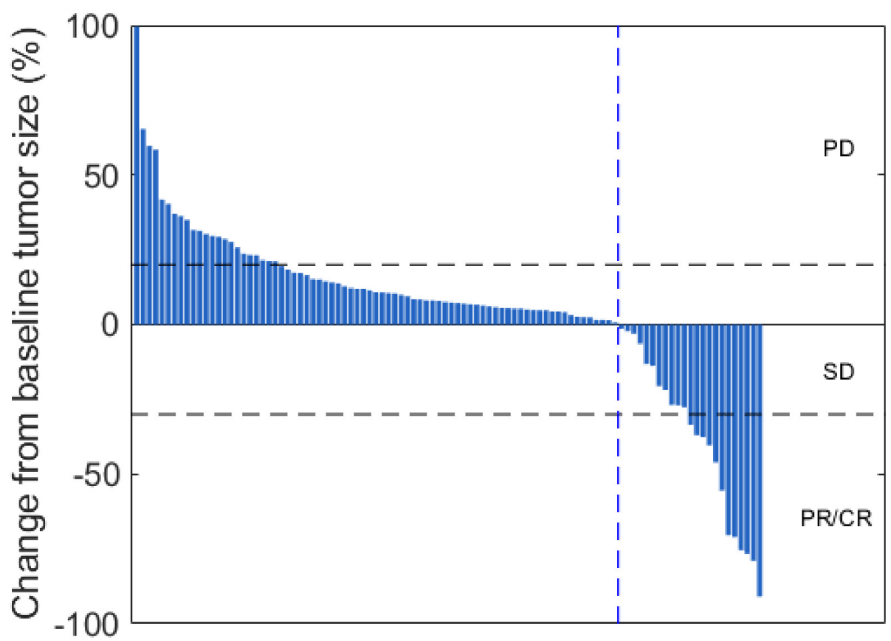
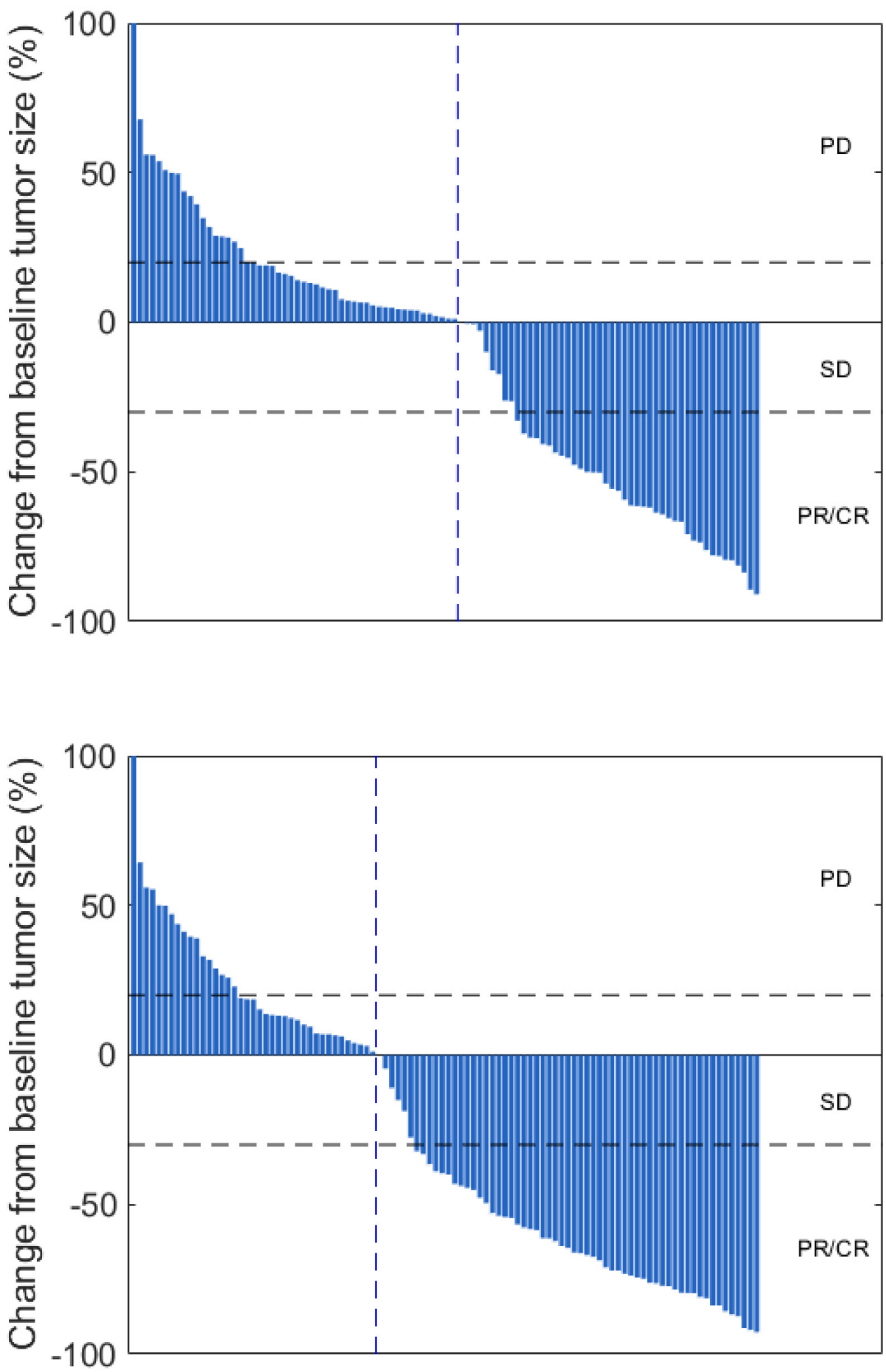

Figure 2 Rate of response (left) and the best overall response (right) in model-predicted tumor diameter of 100 randomly selected virtual patients. Response is assessed by RECIST V.1.1 ${ }^{31}$ in atezolizumab monotherapy (A), nab-paclitaxel group (B), and atezolizumab+nab-paclitaxel group (C). Median (thick lines) and individual (thin line) rate of response are shown in PD (red), SD (purple), and PR/CR (blue) subgroups. CR, complete response; nab, nanoparticle albumin-bound; PD, progressive disease; $\mathrm{PR}$, partial response; $\mathrm{SD}$, stable disease. 

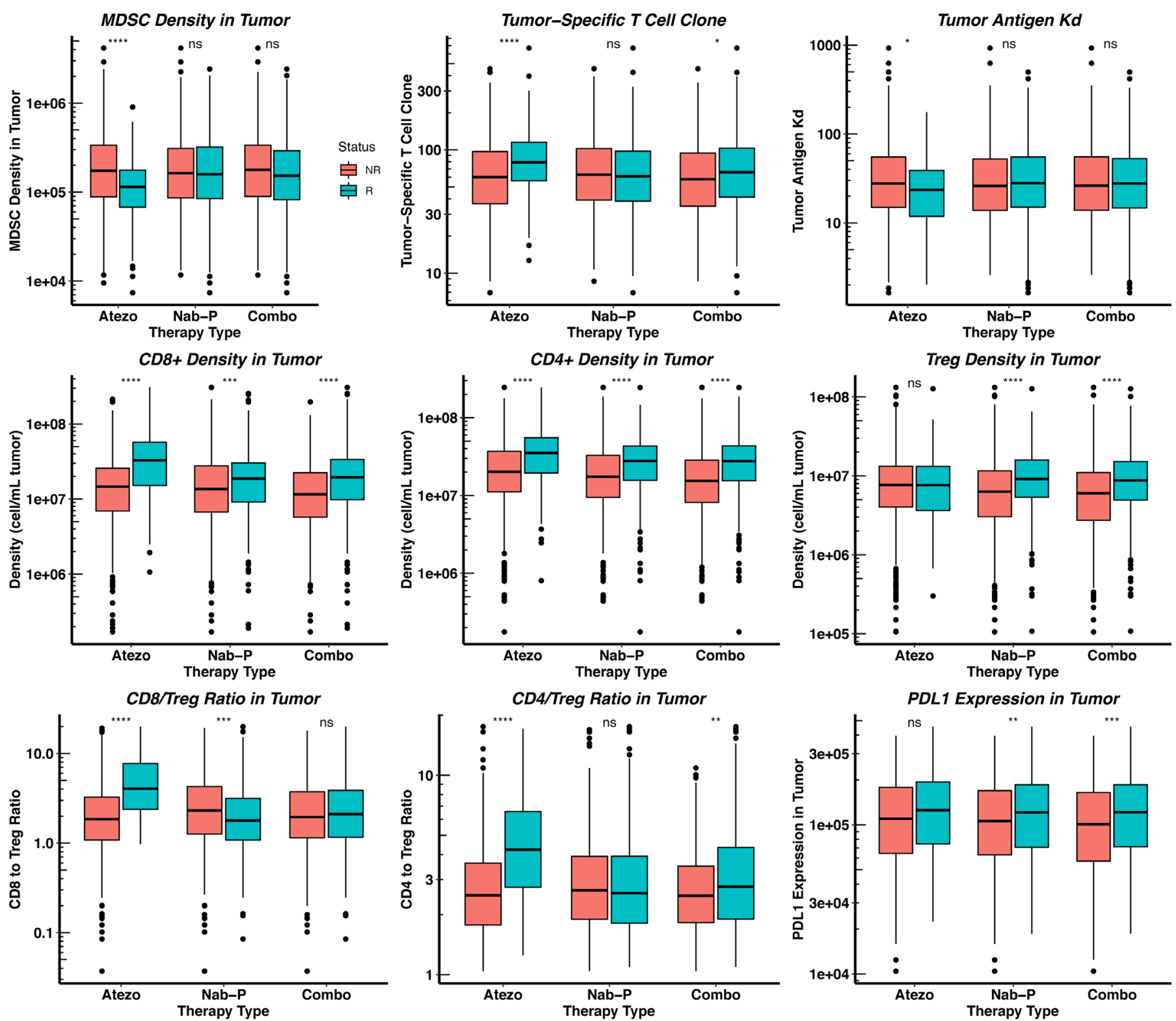

Figure 3 Pretreatment distributions of potential predictive biomarkers in responders and non-responders. Statistical significance is calculated by Wilcoxon test. Atezo, atezolizumab monotherapy $1200 \mathrm{mg}$ every 3 weeks; Combo, atezolizumab $840 \mathrm{mg}$ every 2 weeks+nab-paclitaxel $100 \mathrm{mg} / \mathrm{m}^{2}$ Q3/4W; MDSC, myeloid-derived suppressor cell; Nab-P, nab-paclitaxel 100 $\mathrm{mg} / \mathrm{m}^{2} \mathrm{Q} 3 / 4 \mathrm{~W}$; NR, non-responders; R, responder.

placebo comparator arm, $100 \mathrm{mg} / \mathrm{m}^{2}$ dose is administered days 1,8 , and 15 every 28 days $(\mathrm{Q} 3 / 4 \mathrm{~W})$, and in the experimental arm, a placebo or $840 \mathrm{mg}$ atezolizumab is administered days 1 and 15 every 28 days $(\mathrm{Q} 2 \mathrm{~W})$. As reported by Schmid $e t a l,{ }^{4}$ the ORRs of nab-paclitaxel with placebo and nab-paclitaxel in combination with atezolizumab are $45.9 \%$ and $56.0 \%$, and the DOR for the two regimens are 5.6 and 7.4 months, respectively. To simulate the efficacy of nab-paclitaxel, we introduce a new module to the QSP platform, which incorporates the current knowledge of mechanisms of action of nab-paclitaxel. In summary, the nab-paclitaxel PK is adapted from the published PK model by Chen $e t a l,{ }^{10}$ and its PD is calibrated to experimental and clinical data (online supplemental figure S2). By varying the $\mathrm{PK} / \mathrm{PD}$ parameters in the virtual patient generation, we are able to account for the interindividual variabilities in the drug delivery and resistance.

Similar to the atezolizumab monotherapy, the virtual patient cohort is generated on calibration to perform the virtual clinical trial. As shown in figure $2 \mathrm{~B}$ and $\mathrm{C}$, the rate of response and the best overall response of the randomly selected virtual patients are plotted. The tumor dynamics and the shape of the waterfall plots also resemble those reported by clinical trials of atezolizumab and nabpaclitaxel. ${ }^{37-39}$ To better compare the model predictions of ORR and DOR with clinical results, we generate an efficacy prediction table using a similar format to that reported by Schmid et al. ${ }^{4}$ In table 1, we perform 1000 bootstrap resampling of 450 virtual patients from the whole virtual cohort (900 virtual patients in total) and 
Table 1 Efficacy prediction for the virtual patient cohort generated based on calibrated parameter distribution

\begin{tabular}{|c|c|c|}
\hline Variable & Nab-paclitaxel & $\begin{array}{l}\text { Atezolizumab/ } \\
\text { nab-paclitaxel }\end{array}$ \\
\hline \multicolumn{3}{|l|}{ Response } \\
\hline Number of virtual patients & 450 & 450 \\
\hline \multicolumn{3}{|l|}{ Objective response } \\
\hline No. of patients (median) & 209 & 266 \\
\hline$\%$ of patient (95 bootstrap percentile) & $46.4(41.0-52.0)$ & $59.1(53.2-64.7)$ \\
\hline \multicolumn{3}{|l|}{ Complete response } \\
\hline No. of patients (median) & 9 & 11 \\
\hline$\%$ of patient (95 bootstrap percentile) & $2.0(0.7-3.9)$ & $2.4(0.9-4.4)$ \\
\hline \multicolumn{3}{|l|}{ Partial response } \\
\hline No. of patients (median) & 200 & 255 \\
\hline$\%$ of patient (95 bootstrap percentile) & $44.4(38.9-50.7)$ & $56.7(50.6-62.2)$ \\
\hline \multicolumn{3}{|l|}{ Stable disease } \\
\hline No. of patients (median) & 138 & 106 \\
\hline$\%$ of patient (95 bootstrap percentile) & $30.7(25.7-36.2)$ & $23.6(18.7-28.2)$ \\
\hline \multicolumn{3}{|l|}{ Progressive disease } \\
\hline No. of patients (median) & 103 & 78 \\
\hline$\%$ of patient (95 bootstrap percentile) & $22.9(18.2-27.6)$ & $17.3(13.1-21.6)$ \\
\hline \multicolumn{3}{|l|}{ Duration of response } \\
\hline Number of virtual patients & 209 & 266 \\
\hline $\begin{array}{l}\text { Median duration of response in months } \\
\text { ( } 95 \text { bootstrap percentile) }\end{array}$ & $5.6(5.6-7.5)$ & $7.5(5.6-9.3)$ \\
\hline $\begin{array}{l}\text { No. of virtual patients with ongoing response after } 400 \text { days of } \\
\text { treatment in model simulations (\%) }\end{array}$ & $69(33.2)$ & $87(32.7)$ \\
\hline
\end{tabular}

Bootstrap median and $\mathrm{Cls}$ are reported.

calculate the bootstrap medians and the 95 percentile bootstrap CIs for the endpoint predictions in each treatment regimen. While most of the efficacy predictions overlap with the ranges reported by the clinical trial (table 1; online supplemental figure S2), the complete response rate is underestimated. Although we predict a tumor smaller than $2 \mathrm{~mm}$ as a complete response by assuming limited detectability by imaging modalities, a tumor that is smaller than $2 \mathrm{~mm}$ can be detected in some cases. ${ }^{40}$ Additionally, the inhibitory effect of MDSC, Treg, immune checkpoints, and cytokines on Teff may be overestimated by the model, which together lead to the underestimation of complete tumor eradication.

As shown in figure 3, the distributions of potential predictive biomarkers show different trends in the three treatment regimens. Unlike atezolizumab monotherapy, the pretreatment MDSC density, the number of tumorspecific $\mathrm{T}$ cell clones, neoantigen $\mathrm{K}_{\mathrm{D}}$, and $\mathrm{CD}^{+} /$Treg ratio are not significantly affected by the response status. This prediction suggests that the efficacy of chemotherapy may not depend on the reversal of the inhibition on preexisting Teffs, but on the therapy-induced activation of immune response by newly released tumor antigens. Interestingly, figure 3 shows that the pretreatment $\mathrm{CD}^{+} /$
Treg ratio is significantly lower in the responders of nabpaclitaxel monotherapy, which does not match our expectation. In figure 4, we divide the virtual patient cohort into subgroups by their pretreatment values of selected biomarkers and calculate the corresponding ORR with the 95\% Agresti-Coull CI. The CIs for subgroups based on the $\mathrm{CD}^{+}$and $\mathrm{CD} 4^{+} \mathrm{T}$ cell levels show significant differences in the corresponding response rates, while those for other subgroups overlap. These results suggest that the response to combination therapy of PD-L1 inhibitors and nab-paclitaxel correlates with high $\mathrm{T}$ cell levels, although this was not observed for $\mathrm{CD}^{+} \mathrm{T}$ cells in IMpassion $130 .{ }^{41}{ }^{42}$ In addition, subgroups with high PD-L1 expression and $\mathrm{CD}^{+} /$Treg ratio in the tumor also correspond to notably higher ORRs than those with low PD-L1 expression and $\mathrm{CD} 4^{+} /$Treg ratio.

\section{Performance of potential predictive biomarkers}

The performance of the predictive biomarkers identified previously is investigated using a binary classification model. As shown in figure 5, the sensitivity and 1-specificity values from each cut-off were plotted as receiver operating characteristic (ROC) curves. $\mathrm{CD} 8^{+}$ and $\mathrm{CD} 4^{+} \mathrm{T}$ cell densities have higher areas under curves 


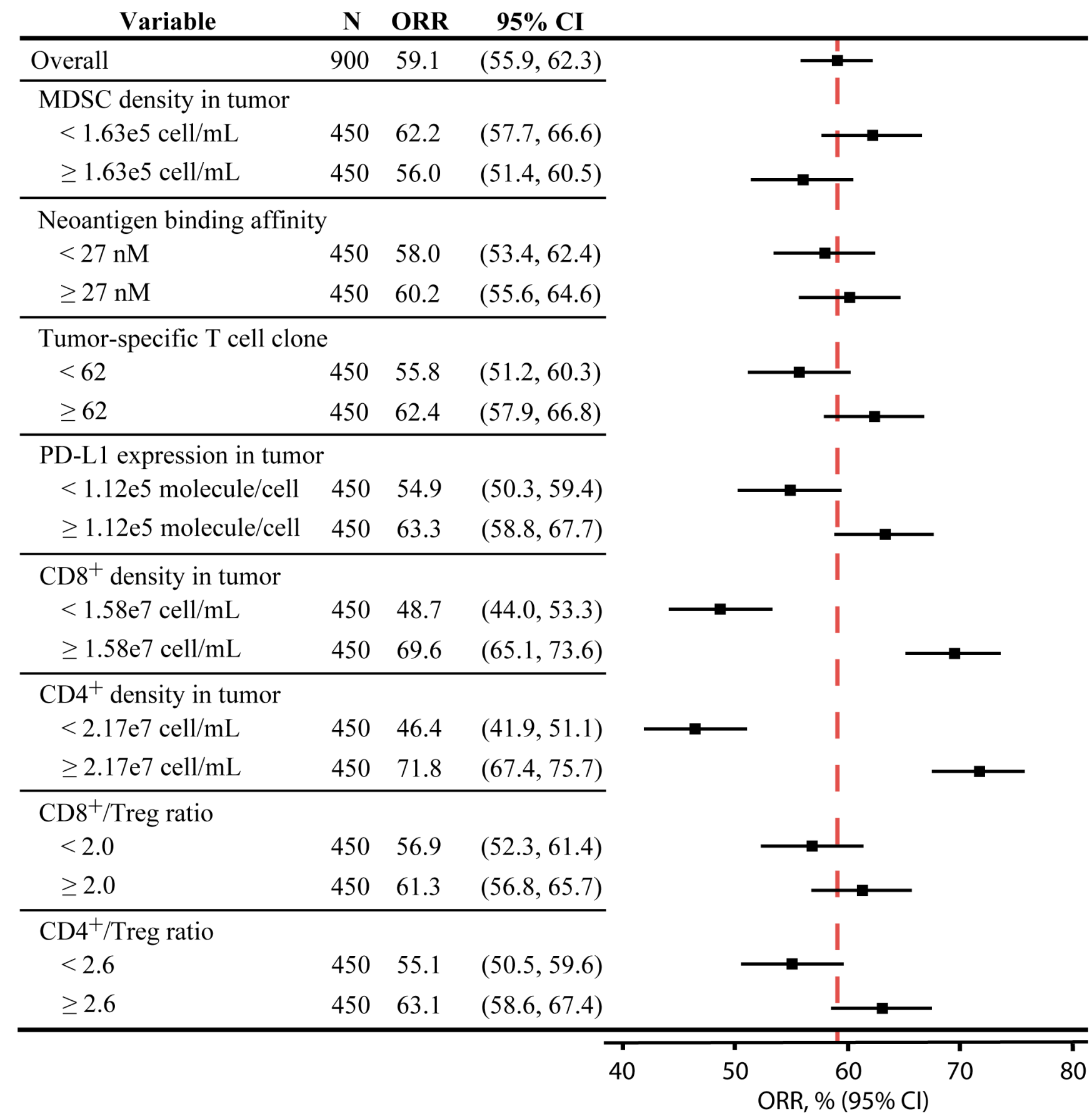

Figure 4 Subgroup analysis of the combination therapy in virtual patient cohort. The total 900 virtual patients are divided into eight subgroups based on the pretreatment values of selected biomarkers, and the objective response rates in each subgroup are calculated with 95\% Agresti-Coull Cls. MDSC, myeloid-derived suppressor cell.

(AUCs) (0.631 and 0.659, respectively) than PD-L1 expression and $\mathrm{CD} 8^{+} /$Treg and $\mathrm{CD} 4^{+} /$Treg ratios $(0.570$, 0.507 , and 0.554 , respectively), further implicating their potential to be predictive biomarkers for this double combination regimen. However, the model-predicted AUCs are not as high as those reported in our previous simulations and clinical analyses for anti-PD-1/PD-L1 monotherapy. ${ }^{2043}$ Furthermore, the dynamics of selected biomarkers are investigated under the three treatment regimens in online supplemental figure S3. The posttreatment (week 8) to pretreatment ratios suggest that either atezolizumab or nab-paclitaxel alone is able to significantly reduce tumor volume and increase both $\mathrm{CD} 8^{+}$and $\mathrm{CD} 4^{+}$densities in tumor within 8 weeks on drug administration. The addition of atezolizumab to nabpaclitaxel further significantly increases tumor volume reduction and $\mathrm{T}$ cell levels, which shows an additive effect of the double combination therapy. Interestingly, the $\mathrm{CD}^{+} /$Treg ratio in the tumor is significantly lowered by nab-paclitaxel at week 8 , which agrees with the in vivo observations and needs to be validated by future experimental or clinical results. ${ }^{44}$ 


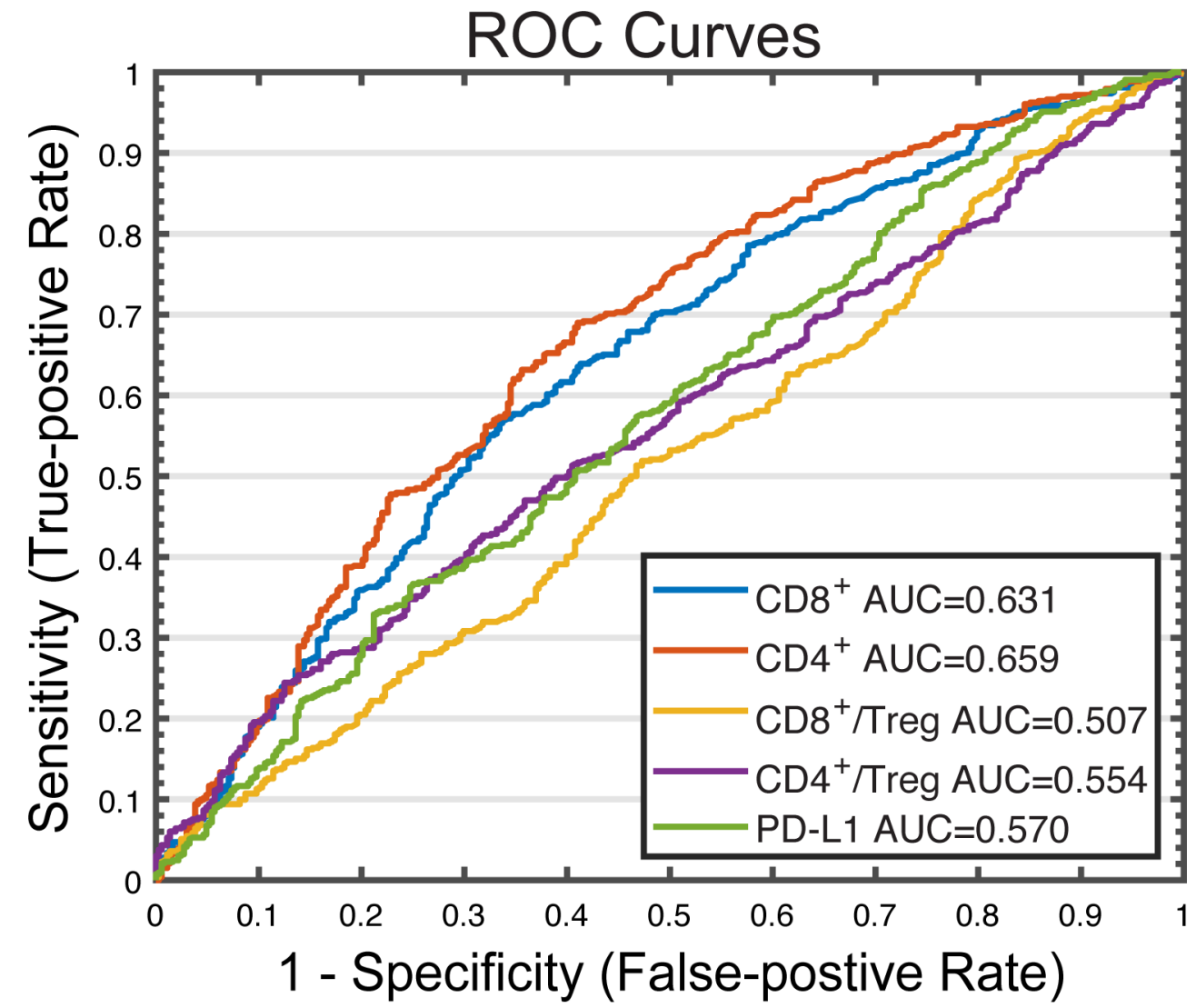

Figure 5 ROC analysis of potential predictive biomarkers in combination therapy. Cut-off values are selected based on the range of PD-L1 molecules on APCS, pretreatment effector T cell density, tumor mutational burden, and Teff to regulatory $\mathrm{T}$ cell ratio. For each cut-off value, response status (R vs NR) is predicted for each virtual patient by comparing the pretreatment amount of the potential predictive biomarker to the cut-off value. Sensitivity (true positive rate) is plotted against 1 - specificity (true negative rate) for each biomarker. APCs, antigen-presenting cells; AUC, areas under curve; NR, non-responders; R, responders; $\mathrm{ROC}$, receiver operating characteristic.

To further explore the effect of the biomarkers on model predictions, we perform the global uncertainty and sensitivity analysis using the calibrated virtual patient distribution. As shown in online supplemental figure S4, 26 parameters are varied in the virtual patient generation, including parameters with fitted range to experimental data and those with estimated range that are then calibrated by the clinical results. Additionally, for a subset of parameters varied in the virtual patient generation, we sort the virtual patients by each parameter in ascending orders and evenly divide them into multiple subgroups. The ORR of each subgroup in the double combination therapy is plotted against the median parameter values in figure 6. As a result, predicted ORRs show a trend of increase as PD-L1 expression, $\mathrm{CD}^{+}$and $\mathrm{CD} 4^{+} \mathrm{T}$ cell densities increase, while ORRs decrease as tumor growth rate and pretreatment tumor size increase.

\section{Sequential therapy simulation}

Now that the present QSP platform is validated by its prediction of the additive effect of atezolizumab to nabpaclitaxel on ORR and DOR (table 1; online supplemental figure S2), we aim to explore its potential for dose optimization. To this end, we perform a series of virtual clinical trials using various doses and schedules of nab-paclitaxel and atezolizumab. Specifically, $840 \mathrm{mg}$ atezolizumab is administered every 2 weeks starting on day 1 , week 2 , or week 4 on reaching the initial tumor diameter, in combination with nab-paclitaxel, and $100 \mathrm{mg} / \mathrm{m}^{2}$ (on days 1,8 , and 15 of a 28-day cycle), $125 \mathrm{mg} / \mathrm{m}^{2}$ (on days 1 and 8 of a 21-day cycle), or $260 \mathrm{mg} / \mathrm{m}^{2}$ (every 3 weeks) of nab-paclitaxel is administered staring on day 1 , week 2, or week 4 (figure 7A-C, online supplemental S5A-C). These selected dose schedules have been used in clinical trials of combination therapies of nab-paclitaxel and other therapeutic agents, which are adopted here to avoid potential toxicity concerns. ${ }^{7}$ The median tumor volume, $\mathrm{CD}^{+} \mathrm{T}$ cell level, and Treg density, $\mathrm{CD} 8^{+} /$Treg, and $\mathrm{CD}^{+} /$Treg ratios in the tumor at week 8 are reported with the ORR for each combination of the treatment regimens in figure 7 and online supplemental figure S5. Although $260 \mathrm{mg} / \mathrm{m}^{2}$ nab-paclitaxel results in the highest predicted ORR in the concurrent therapy (online supplemental figure S5), more frequent dosing with $100 \mathrm{mg} / \mathrm{m}^{2}$ nab-paclitaxel leads to the highest median tumor volume reduction and $\mathrm{CD} 8^{+} \mathrm{T}$ cell level at week 8 (figure 7). Notably, simulating concurrent therapy with atezolizumab and $260 \mathrm{mg} / \mathrm{m}^{2}$ nab-paclitaxel shows high Treg density in the tumor (figure 7 ), which corresponds 

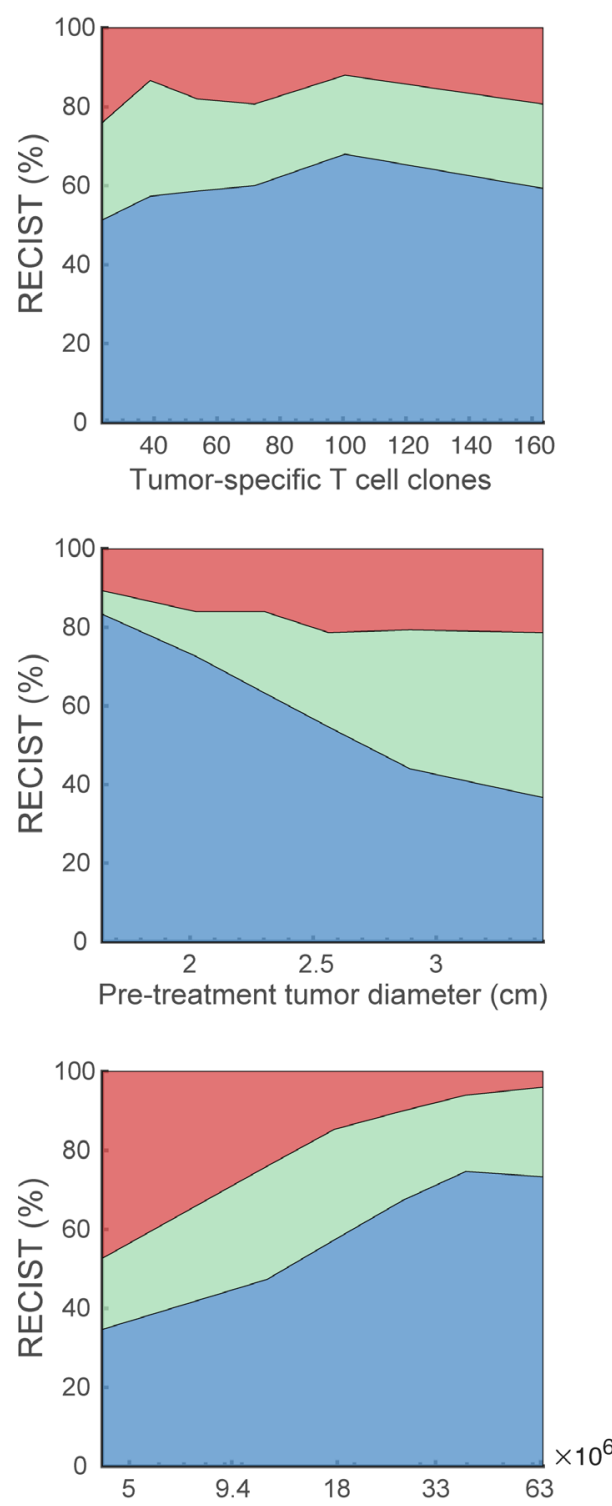

Pre-treatment CD4 T cell density (cell/mL)
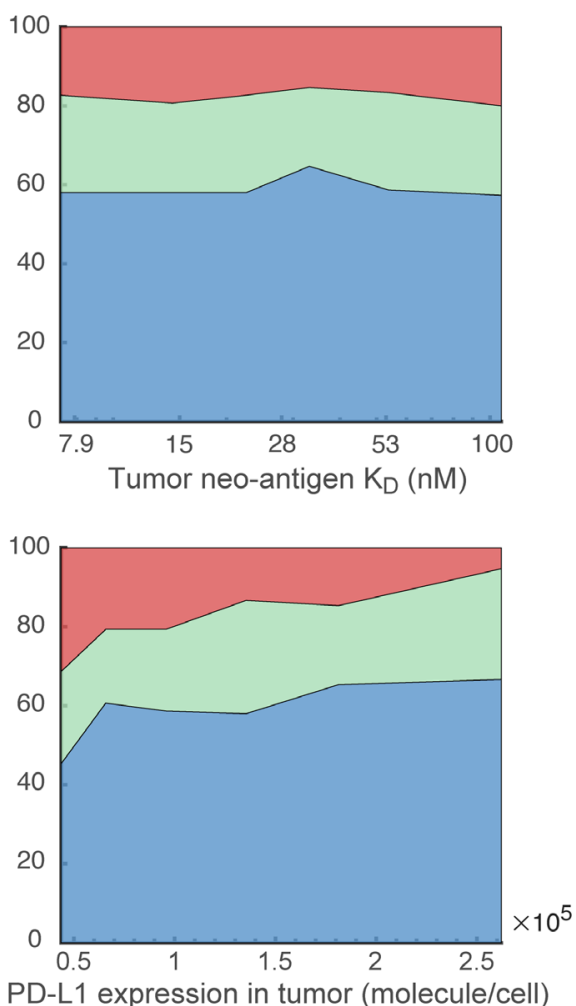

PD-L1 expression in tumor (molecule/cell)

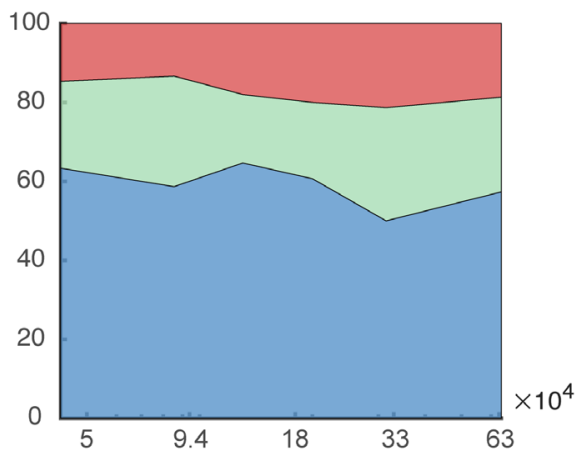

Pre-treatment MDSC density (cell/mL)
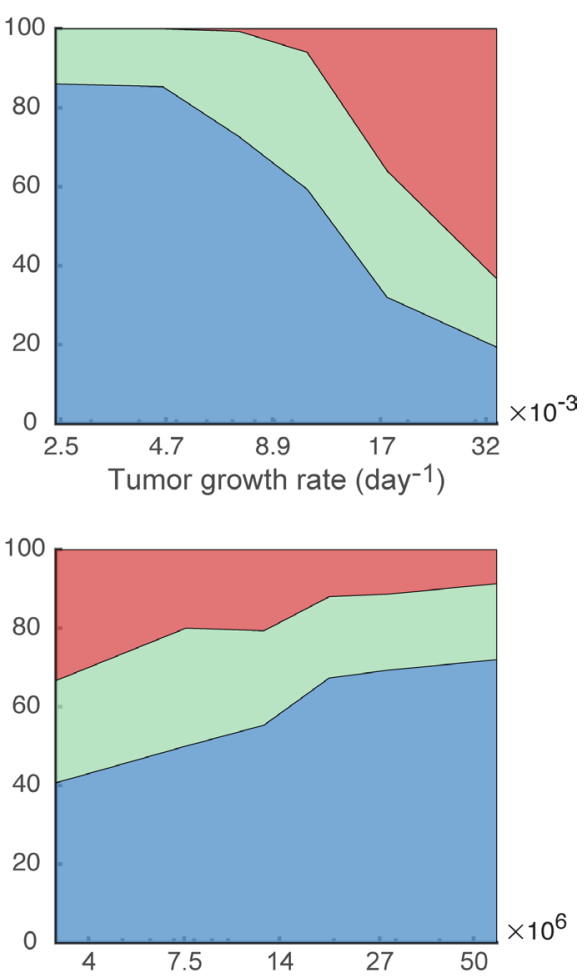

Pre-treatment CD8 T cell density (cell/mL)

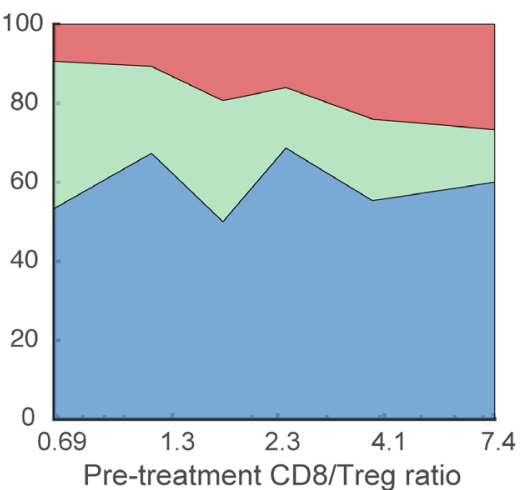

Figure 6 Effects of parameters on objective response. For each parameter of interest, 900 virtual patients are sorted by the parameter values in ascending order and evenly divided into nine subgroups. The response status of each subgroup in the combination therapy is plotted against the corresponding median parameter values. MDSC, myeloid-derived suppressor cells.

to the lowest $\mathrm{CD}^{+} /$Treg and $\mathrm{CD} 4^{+} /$Treg ratios among the combination regimens (online supplemental figure S5). This upregulation of Treg density was also observed in a preclinical in vivo study of murine TNBC treated with paclitaxel. ${ }^{44}$ Overall, the virtual clinical trials suggest that concurrent therapies of atezolizumab and nab-paclitaxel lead to better response than sequential therapies, and the model predictions agree with the clinical observation that weekly $100 \mathrm{mg} / \mathrm{m}^{2}$ nab-paclitaxel shows similar efficacy to $300 \mathrm{mg} / \mathrm{m} 2$ every 3 weeks dosing but with reduced toxicity. ${ }^{45}$

\section{DISCUSSION}

In this study, we introduce multiple modifications to our previously developed QSP platform, including a new nab-paclitaxel module and modified cancer, $\mathrm{T}$ cell, and checkpoint modules. ${ }^{204647}$ The addition of nab-paclitaxel begins our attempt to make efficacy prediction for combination therapies involving checkpoint inhibitors and chemotherapeutic agents. While nab-paclitaxel has been approved for the treatment of metastatic breast cancer, its efficacy when combined with other types of therapeutic agents is still under investigation. Here, we implement all the known effects of nab-paclitaxel on TNBC cell lines, including its cytotoxic, angiogenic, and antiangiogenic activities, as reported by in vivo preclinical and clinical observations. ${ }^{12-15}$ As we incorporate all the mechanisms of action into the model, we are able to investigate the overall effect of nab-paclitaxel on tumor dynamics. In addition to the new module, the available clinical data of $\mathrm{T}$ cell levels in patients with TNBC allow us to expand the $\mathrm{T}$ cell module to better describe the dynamics of naive $\mathrm{T}$ 

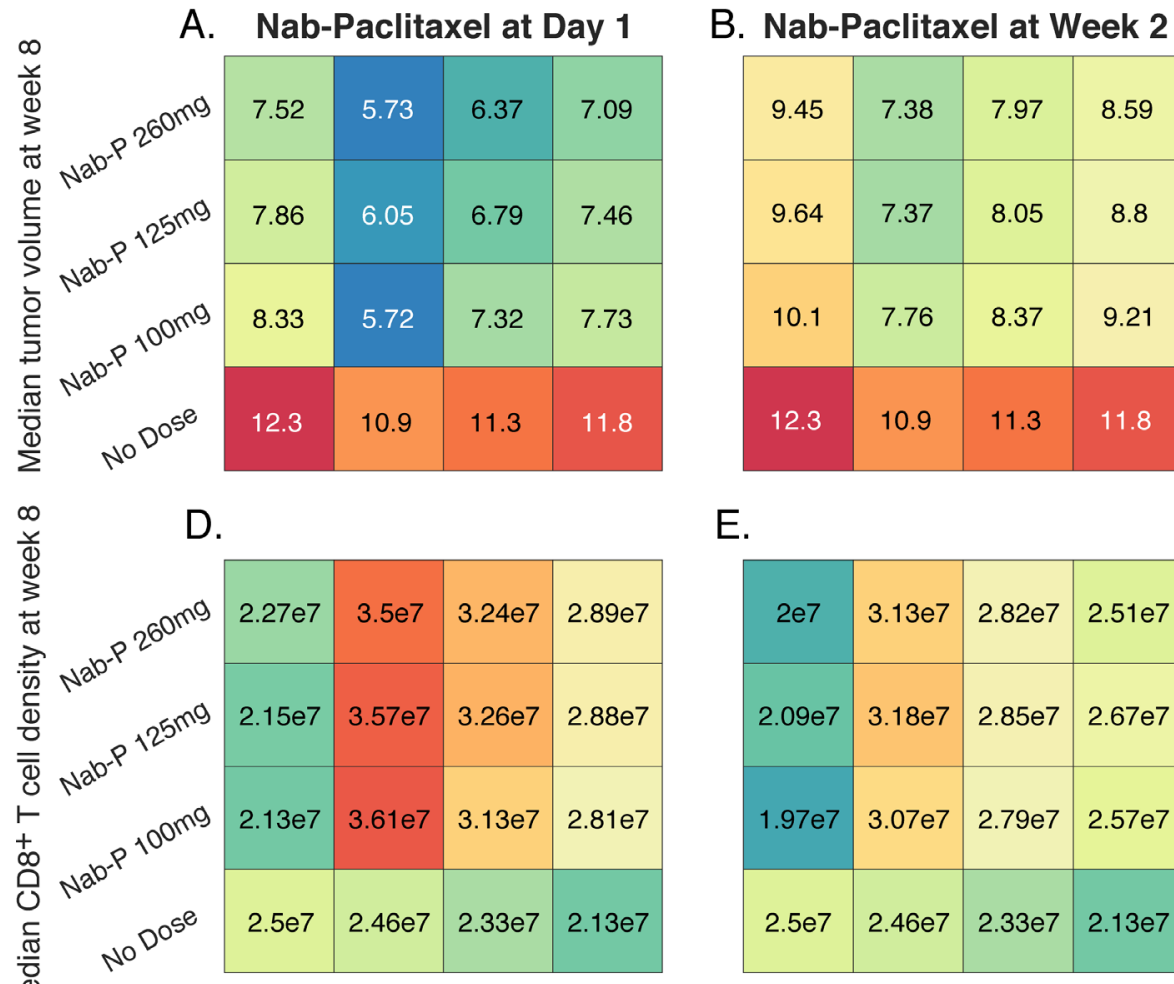

E.

G.

$\mathrm{H}$.

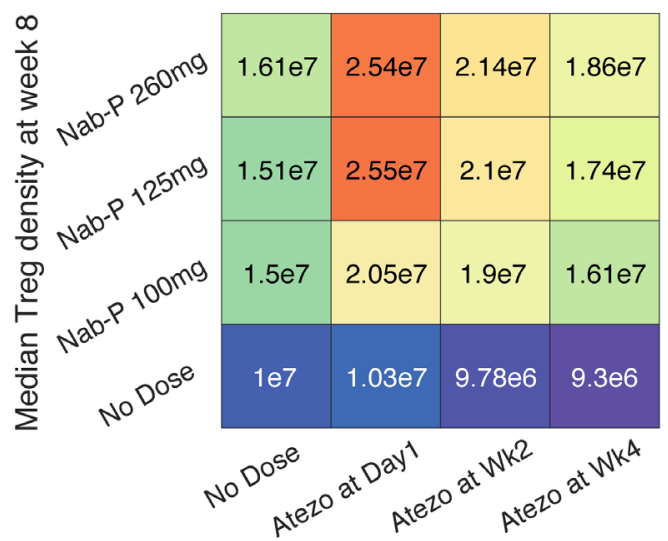

\begin{tabular}{|c|c|c|c|}
\hline 9.45 & 7.38 & 7.97 & 8.59 \\
\hline 9.64 & 7.37 & 8.05 & 8.8 \\
\hline 10.1 & 7.76 & 8.37 & 9.21 \\
\hline 12.3 & 10.9 & 11.3 & 11.8 \\
\hline
\end{tabular}

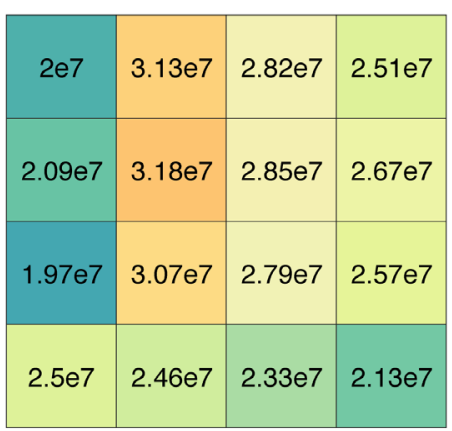

C. Nab-Paclitaxel at Week 4

\begin{tabular}{|c|c|c|c|}
\hline 10.8 & 8.65 & 9.6 & 10 \\
\hline 11.4 & 8.82 & 9.32 & 10.4 \\
\hline 11.4 & 8.91 & 9.55 & 10.6 \\
\hline 12.3 & 10.9 & 11.3 & 11.8 \\
\hline
\end{tabular}

F.

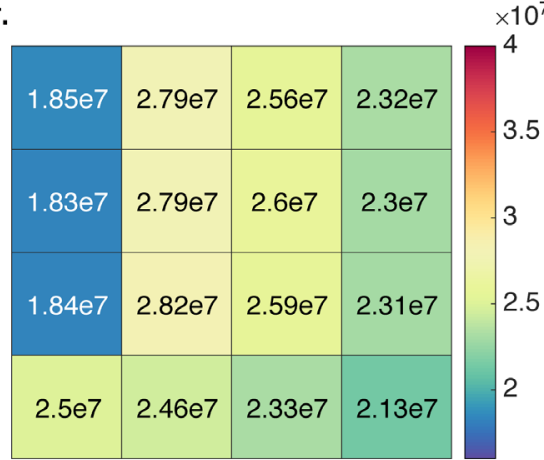

I.

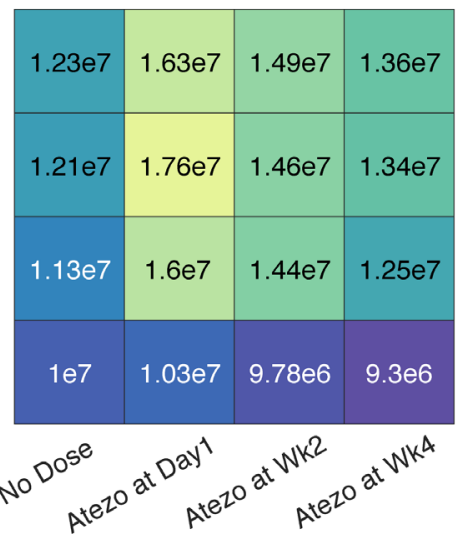

Figure 7 Model simulation of sequential therapies using various nab-paclitaxel doses and schedules. Top row (A-C) represents the median tumor volume after 8 weeks of each dose regimen; middle row $(D-F)$ represents the median $C D 8^{+} T$ cell density at week 8; and bottom row (G-I) represents the median Treg density in the tumor at week 8. Administration of nab-paclitaxel starts on day $1(A, D, G)$, week $2(B, E, H)$, and week $4(C, F, I)$ on reaching initial tumor diameter.

cells, $\mathrm{CD}^{+}$helper $\mathrm{T}$ cells, and the interactions within the $\mathrm{T}$ cell subsets, such as Th to Treg transdifferentiation. As shown in figure $3, \mathrm{CD} 8^{+}, \mathrm{CD} 4^{+}$, and Treg densities in the tumor all fall within the physiologically realistic ranges reported by clinical measurements. ${ }^{27-29}$ Furthermore, the upregulation of PD-L1 expression by interferon gamma (IFN- $\gamma$ ) in the tumor is implemented into the checkpoint module to account for the negative feedback mechanism that follows the tumor infiltration of lymphocytes. ${ }^{48}$ This mechanism allows us to better predict the dynamics of PD-L1 expression during the therapy and how it is associated with the response to the combination therapy.

In this retrospective clinical trial analysis using our proposed QSP platform, we demonstrate its potential for making efficacy predictions of checkpoint inhibitors and chemotherapeutic agents by conducting virtual clinical trials. When the distributions of model parameters are calibrated by data from the two monotherapies, the model-predicted efficacy of atezolizumab in combination with nab-paclitaxel overlaps with the experimental arm of the IMpassion130 trial. ${ }^{4}$ However, the comparisons between our model predictions and the clinical results also reflect the limitations of the present model. Particularly, the efficacy prediction for atezolizumab monotherapy shows a shift from progressive disease to stable disease. This discrepancy is due to the complexity of the immune system and the clinical settings that cannot be entirely captured or reproduced by model simulations. 
Using model simulations, we identify the objective response status of the virtual patients by their best overall response assuming a spherical tumor. In other words, virtual patients with a reduction of tumor size more than $30 \%$ at any time point of the simulation are considered as partial or complete responders; those with an increase of tumor size more than $20 \%$ within 8 weeks on the dose administration are considered to have progressive disease; and the rest are considered to have stable disease. ${ }^{31}$ In reality, the characterization of response status is also impacted by other conditions, such as patients' survival, new metastatic lesions, the definition of tumor burden as the sum of largest diameters, and resolution of the imaging modalities, all of which are likely to cause a deviation from model predictions.

In the biomarker analysis, the model first confirms that the number of tumor-specific $\mathrm{T}$ cell clones (as a measure of tumor mutational burden) and $\mathrm{T}$ cell densities in the tumor are associated with response status in single-agent PD-L1 blockade therapies. ${ }^{64-36}$ For the combination of atezolizumab and nab-paclitaxel, the model confirms that subgroups with high PD-L1 expression have higher ORRs, even though the increase is not as significant as reported by the trial. Besides, the model identifies that $\mathrm{CD} 8^{+}$and $\mathrm{CD}^{+} \mathrm{T}$ cell levels are the best two predictive biomarkers due to their significantly higher medians in responders and the significantly higher ORR in subgroups with high levels of T cells (figures 3 and 4). Although similar correlations are observed in neoadjuvant chemotherapies using other cytotoxic agents, ${ }^{41}{ }^{42}$ the performance of these biomarkers needs to be explored in combination treatments. Nonetheless, there exist discrepancies between model-predicted biomarkers and clinical observations in chemotherapy. As shown in figure 3, the pretreatment $\mathrm{CD}^{+} /$Treg ratio is significantly lower in the responders of nab-paclitaxel monotherapy, but this correlation has not been confirmed by clinical studies. In fact, pathological complete response rate is reported to be significantly higher in patients with early-stage TNBC in high-CD8 ${ }^{+}$/ FoxP3 $^{+}$ratio group during FEC100 treatment. ${ }^{49}$ Although this discrepancy is possibly due to the differences in treatment regimens and immunobiology between early-stage and metastatic TNBC, the relationship between $\mathrm{CD}^{+} /$ Treg ratio and response status in nab-paclitaxel treatment remains to be confirmed by clinical studies.

Furthermore, due to the promising synergistic effect of sequential therapies using checkpoint inhibitors and other therapeutic agents in multiple cancer types, we performed a series of in silico clinical trials to investigate the optimal dose schedule of atezolizumab and nabpaclitaxel for TNBC. The results suggest that concurrent therapies result in higher ORRs and T cell densities than sequential therapies. In addition, although $260 \mathrm{mg} / \mathrm{m}^{2}$ every 3 weeks nab-paclitaxel corresponds to the highest ORR, more frequent doses with $100 \mathrm{mg} / \mathrm{m}^{2} \mathrm{Q} 3 / 4 \mathrm{~W}$ (on days 1,8 , and 15 of a 28-day cycle) have similar ORR and higher $\mathrm{CD}^{+} \mathrm{T}$ cell levels with potentially less toxicity. ${ }^{50}$ Clinical data from other trials involving checkpoint inhibitors and chemotherapy in TNBC would be valuable for model calibration and validation, such as IMpassion132 and KEYNOTE-355. Notably, the simulated dose regimens are adopted from the existing clinical trial protocols whose safety has been tested clinically to avoid potential toxicity concerns. Future incorporation of toxicity prediction into the QSP platform will allow optimization of dose intensity and frequency for atezolizumab. In a recently published study, Ma et a $\tilde{l}^{\tilde{1}}$ suggest cytokine release syndrome, which is a common dose-dependent adverse event caused by cancer immunotherapy, to be predicted by mechanistic modeling of interleukin-6 expression. However, this method requires mechanistic understandings of immune-related adverse effects and patient-level data from clinical trials for model implementation, which is currently unavailable for atezolizumab.

Overall, the predictive power of virtual clinical trials depends on three factors: translation of molecular and cellular mechanisms into model reactions, parameter estimation using experimental data, and calibration of the virtual patient distribution. First, the QSP model aims to incorporate the current knowledge of molecular and cellular mechanisms to make accurate predictions based on preclinical observations. However, the phenotypic observations may not reflect the true mechanisms behind them. In many cases, hypotheses have to be made to incorporate the observed dynamics into the model. In addition, the degrees of mechanistic detail are limited by the scope of the model. For example, the development of chemoresistance is known to be related to cytokines, membrane proteins, gene expression changes, and many other pathways in cancer cells. ${ }^{52}$ Since it is not pragmatic to incorporate all known mechanisms into the model in every detail, a constant effort to optimize the level of model complexity is essential. Second, the parameter estimation remains one of the major challenges when building QSP models, given their high levels of complexity. In this study, most of the parameters are estimated using experimental data of breast cancer, as our goal is to simulate tumor dynamics in patients with advanced TNBC. However, a number of issues remain to be explored: how to adjust parameters for spatial heterogeneity or for the tumor that metastasized from its primary tumor site? How are parameters derived from animal data translated to humans? How to account for potential covariance between the model parameters in the virtual patient generation (eg, between body surface area and PK parameters)? Third, the distributions of parameters varied in the virtual patient generation are calibrated by clinical results, which can be biased toward the inclusion criteria of the trial. Notably, there are a number of methods for the virtual patient generation with different algorithms to address potential biases. ${ }^{21-25}$ Here, we use the methods that are similar to the recently published studies..$^{53} 54$ The optimal techniques for virtual patient generation based on the availability of clinical data is an active area of research that is undergoing rapid development. 
Correction notice This article has been corrected since it first published. The provenance and peer review statement has been included.

Twitter Hanwen Wang @HanwenWang95, Leisha A Emens @EmensLeisha and Aleksander S Popel @HopkinsPopelLab

Acknowledgements The authors would like to thank Qingqing Yin for her helpful comments on the statistical analyses that improved the manuscript.

Contributors ASP, LAE, and HW designed and planned the project. HW, HM, and RJS built the model. HW modified the model, performed all simulations, analyzed the simulation data, and prepared a draft of the manuscript. ASP and LAE critically revised the manuscript. All authors have read and approved the final manuscript.

Funding Supported by NIH grant R01CA138264.

Competing interests None declared.

Patient consent for publication Not required.

Provenance and peer review Not commissioned; externally peer reviewed.

Data availability statement The authors confirm that the data supporting the findings of this study are available within the article and the supplementary material. MATLAB scripts for model generation and in silico clinical trials are publicly available at https://doi.org/10.5281/zenodo.4437288 and on GitHub (https://github.com/popellab/qspio-tnbc).

Supplemental material This content has been supplied by the author(s). It has not been vetted by BMJ Publishing Group Limited (BMJ) and may not have been peer-reviewed. Any opinions or recommendations discussed are solely those of the author(s) and are not endorsed by BMJ. BMJ disclaims all liability and responsibility arising from any reliance placed on the content. Where the content includes any translated material, BMJ does not warrant the accuracy and reliability of the translations (including but not limited to local regulations, clinical guidelines, terminology, drug names and drug dosages), and is not responsible for any error and/or omissions arising from translation and adaptation or otherwise.

Open access This is an open access article distributed in accordance with the Creative Commons Attribution Non Commercial (CC BY-NC 4.0) license, which permits others to distribute, remix, adapt, build upon this work non-commercially, and license their derivative works on different terms, provided the original work is properly cited, appropriate credit is given, any changes made indicated, and the use is non-commercial. See http://creativecommons.org/licenses/by-nc/4.0/.

\section{ORCID iDs}

Hanwen Wang http://orcid.org/0000-0001-5480-431X

Huilin Ma http://orcid.org/0000-0003-4238-9475

Aleksander S Popel http://orcid.org/0000-0002-6706-9235

\section{REFERENCES}

1 Gradishar WJ, Anderson BO, Balassanian R, et al. NCCN guidelines insights: breast cancer, version 1.2017. J Natl Compr Canc Netw 2017;15.

2 Emens LA, Ascierto PA, Darcy PK, et al. Cancer immunotherapy: opportunities and challenges in the rapidly evolving clinical landscape. Eur J Cancer 2017;81:116-29.

3 Schmid P, Rugo HS, Adams S, et al. Atezolizumab plus nabpaclitaxel as first-line treatment for unresectable, locally advanced or metastatic triple-negative breast cancer (IMpassion130): updated efficacy results from a randomised, double-blind, placebo-controlled, phase 3 trial. Lancet Oncol 2020;21:44-59.

4 Schmid P, Adams S, Rugo HS, et al. Atezolizumab and nabpaclitaxel in advanced triple-negative breast cancer. $N$ Engl J Med 2018;379:2108-21.

5 Emens LA. Breast cancer immunotherapy: facts and hopes. Clin Cancer Res 2018;24:511-20.

6 Marra A, Viale G, Curigliano G. Recent advances in triple negative breast cancer: the immunotherapy era. BMC Med 2019;17:90.

7 Brufsky A. nab-Paclitaxel for the treatment of breast cancer: an update across treatment settings. Exp Hematol Oncol 2017;6:7.

8 Di Costanzo F, Gasperoni S, Rotella V, et al. Targeted delivery of albumin bound paclitaxel in the treatment of advanced breast cancer. Onco Targets Ther 2009;2:179-88.

9 Zhu A, Yuan P, Du F, et al. Sparc overexpression in primary tumors correlates with disease recurrence and overall survival in patients with triple negative breast cancer. Oncotarget 2016;7:76628-34.

10 Chen N, Li Y, Ye Y, et al. Pharmacokinetics and pharmacodynamics of nab-paclitaxel in patients with solid tumors: disposition kinetics and pharmacology distinct from solvent-based paclitaxel. J Clin Pharmacol 2014;54:1097-107.

11 Desai N, Trieu V, Yao Z, et al. Increased antitumor activity, intratumor paclitaxel concentrations, and endothelial cell transport of cremophor-free, albumin-bound paclitaxel, ABI-007, compared with cremophor-based paclitaxel. Clin Cancer Res 2006;12:1317-24.

12 Volk LD, Flister MJ, Bivens CM, et al. Nab-Paclitaxel efficacy in the orthotopic model of human breast cancer is significantly enhanced by concurrent anti-vascular endothelial growth factor a therapy. Neoplasia 2008;10:613-23.

13 Mollard S, Ciccolini J, Imbs D-C, et al. Model driven optimization of antiangiogenics + cytotoxics combination: application to breast cancer mice treated with bevacizumab + paclitaxel doublet leads to reduced tumor growth and fewer metastasis. Oncotarget 2017;8:23087-98.

14 Volk-Draper LD, Rajput S, Hall KL, et al. Novel model for basaloid triple-negative breast cancer: behavior in vivo and response to therapy. Neoplasia 2012;14:926-42.

15 Trieu V, Ran S, Desai N. Investigation of chemotherapy-induced tumor angiogenesis: rationale for combination of nab-paclitaxel with anti-VEGF therapy. Cancer Research 2007;67:4636.

16 Cyprian FS, Akhtar S, Gatalica Z, et al. Targeted immunotherapy with a checkpoint inhibitor in combination with chemotherapy: a new clinical paradigm in the treatment of triple-negative breast cancer. Bosn J Basic Med Sci 2019;19:227-33.

17 Gong C, Milberg O, Wang B, et al. A computational multiscale agent-based model for simulating spatio-temporal tumour immune response to PD1 and PDL1 inhibition. J $R$ Soc Interface 2017; 14:20170320.

18 Betts A, Haddish-Berhane N, Shah DK, et al. A Translational Quantitative Systems Pharmacology Model for CD3 Bispecific Molecules: Application to Quantify T Cell-Mediated Tumor Cell Killing by P-Cadherin LP DART ${ }^{\circledR}$. Aaps J 2019;21:66.

19 Hardiansyah D, Ng CM. Quantitative systems pharmacology model of chimeric antigen receptor T-cell therapy. Clin Trans/ Sci 2019;12:343-9.

20 Wang $\mathrm{H}$, Sové RJ, Jafarnejad $\mathrm{M}$, et al. Conducting a virtual clinical trial in HER2-negative breast cancer using a quantitative systems pharmacology model with an epigenetic modulator and immune checkpoint inhibitors. Front Bioeng Biotechnol 2020;8:141.

21 Allen RJ, Rieger TR, Musante CJ. Efficient generation and selection of virtual populations in quantitative systems pharmacology models. CPT Pharmacometrics Syst Pharmacol 2016;5:140-6.

22 Rieger TR, Allen RJ, Bystricky L, et al. Improving the generation and selection of virtual populations in quantitative systems pharmacology models. Prog Biophys Mol Biol 2018;139:15-22.

23 Chelliah V, Lazarou G, Bhatnagar S, et al. Quantitative systems pharmacology approaches for Immuno-Oncology: adding virtual patients to the development paradigm. Clin Pharmacol Ther 2020. doi:10.1002/cpt.1987. [Epub ahead of print: 19 Jul 2020].

24 Niederer SA, Aboelkassem Y, Cantwell CD, et al. Creation and application of virtual patient cohorts of heart models. Philos Trans $A$ Math Phys Eng Sci 2020;378:20190558.

25 Sinisi S, Alimguzhin V, Mancini T, et al. Complete populations of virtual patients for in silico clinical trials. Bioinformatics 2020. doi:10.1093/bioinformatics/btaa1026. [Epub ahead of print: $16 \mathrm{Dec}$ 2020].

26 Pappalardo F, Russo G, Tshinanu FM, et al. In silico clinical trials: concepts and early adoptions. Brief Bioinform 2019;20:1699-708.

27 Cimino-Mathews A, Thompson E, Taube JM, et al. Pd-L1 (B7-H1) expression and the immune tumor microenvironment in primary and metastatic breast carcinomas. Hum Pathol 2016;47:52-63.

28 Berben L, Wildiers H, Marcelis L, et al. Computerised scoring protocol for identification and quantification of different immune cell populations in breast tumour regions by the use of QuPath software. Histopathology 2020;77:79-91.

$29 \mathrm{Mi} \mathrm{H}$, Gong C, Sulam J, et al. Digital pathology analysis quantifies spatial heterogeneity of CD3, CD4, CD8, CD20, and FOXP3 immune markers in triple-negative breast cancer. Front Physiol 2020;11:583333.

30 Marino S, Hogue IB, Ray CJ, et al. A methodology for performing global uncertainty and sensitivity analysis in systems biology. $J$ Theor Biol 2008;254:178-96.

31 Eisenhauer EA, Therasse P, Bogaerts J, et al. New response evaluation criteria in solid tumours: revised RECIST guideline (version 1.1). Eur J Cancer 2009;45:228-47.

32 Kassambara A. ggpubr: 'ggplot2' Based Publication Ready Plots. R package version 0.2.2, 2019. Available: https://CRAN.R-project.org/ package $=$ ggpubr

33 Emens LA, Cruz C, Eder JP, et al. Long-Term clinical outcomes and biomarker analyses of Atezolizumab therapy for patients with 
metastatic triple-negative breast cancer: a phase 1 study. JAMA Oncol 2019;5:74-82.

34 Cocco S, Piezzo M, Calabrese A, et al. Biomarkers in triple-negative breast cancer: state-of-the-art and future perspectives. Int $\mathrm{J} \mathrm{Mol} \mathrm{Sci}$ 2020;21. doi:10.3390/ijms21134579. [Epub ahead of print: 27 Jun 2020].

35 Vafaizadeh V, Barekati Z. Immuno-Oncology biomarkers for personalized immunotherapy in breast cancer. Front Cell Dev Biol 2020;8:162.

36 Osipov A, Lim SJ, Popovic A, et al. Tumor Mutational Burden, Toxicity, and Response of Immune Checkpoint Inhibitors Targeting PD(L)1, CTLA-4, and Combination: A Meta-regression Analysis. Clin Cancer Res 2020;26:4842-51.

37 Iwata H, Inoue K, Kaneko K, et al. Subgroup analysis of Japanese patients in a phase 3 study of atezolizumab in advanced triplenegative breast cancer (IMpassion130). Jpn J Clin Oncol 2019;49:1083-91.

38 Adams S, Diamond JR, Hamilton E, et al. Atezolizumab plus nabpaclitaxel in the treatment of metastatic triple-negative breast cancer with 2-year survival follow-up: a phase 1B clinical trial. JAMA Oncol 2019;5:334-42.

39 Schettini F, Giuliano M, De Placido S, et al. Nab-Paclitaxel for the treatment of triple-negative breast cancer: rationale, clinical data and future perspectives. Cancer Treat Rev 2016;50:129-41.

40 Erdi YE. Limits of tumor detectability in nuclear medicine and PET. Mol Imaging Radionucl Ther 2012;21:23-8.

41 García-Teijido P, Cabal ML, Fernández IP, et al. Tumor-Infiltrating lymphocytes in triple negative breast cancer: the future of immune targeting. Clin Med Insights Oncol 2016;10:31-9.

42 Planes-Laine G, Rochigneux P, Bertucci F, et al. Pd-1/Pd-L1 targeting in breast cancer: the first clinical evidences are emerging. A literature review. Cancers 2019;11. doi:10.3390/cancers11071033. [Epub ahead of print: 2207 2019].

43 Lu S, Stein JE, Rimm DL, et al. Comparison of biomarker modalities for predicting response to PD-1/PD-L1 checkpoint blockade: a systematic review and meta-analysis. JAMA Oncol 2019. doi:10.1001/jamaoncol.2019.1549. [Epub ahead of print: $18 \mathrm{Jul}$ 2019].
44 Samanta D, Park Y, Ni X, et al. Chemotherapy induces enrichment of CD47(+)/CD73(+)/PDL1(+) immune evasive triple-negative breast cancer cells. Proc Natl Acad Sci U S A 2018;115:E1239-48.

45 Gradishar WJ, Krasnojon D, Cheporov S, et al. Significantly longer progression-free survival with nab-paclitaxel compared with docetaxel as first-line therapy for metastatic breast cancer. J Clin Oncol 2009;27:3611-9.

46 Jafarnejad M, Gong C, Gabrielson E, et al. A computational model of neoadjuvant PD-1 inhibition in non-small cell lung cancer. Aaps $J$ 2019;21:79.

47 Sové RJ, Jafarnejad M, Zhao C, et al. QSP-IO: a quantitative systems pharmacology toolbox for mechanistic multiscale modeling for Immuno-Oncology applications. CPT Pharmacometrics Syst Pharmacol 2020;9:484-97.

48 Sabatier R, Finetti P, Mamessier E, et al. Prognostic and predictive value of PDL1 expression in breast cancer. Oncotarget 2015;6:5449-64.

49 Goto W, Kashiwagi S, Asano Y, et al. Predictive value of improvement in the immune tumour microenvironment in patients with breast cancer treated with neoadjuvant chemotherapy. ESMO Open 2018;3:e000305

50 Martín M. Nab-Paclitaxel dose and schedule in breast cancer. Breast Cancer Res 2015:17:81.

$51 \mathrm{Ma} \mathrm{H}$, Pilvankar M, Wang J, et al. Quantitative systems pharmacology modeling of PBMC-Humanized mouse to facilitate preclinical Immuno-oncology drug development. ACS Pharmacology \& Translational Science 2020.

52 Nedeljković M, Damjanović A. Mechanisms of chemotherapy resistance in triple-negative breast Cancer-How we can rise to the challenge. Cells 2019;8. doi:10.3390/cells8090957. [Epub ahead of print: 2208 2019].

53 Kozłowska E, Vallius T, Hynninen J, et al. Virtual clinical trials identify effective combination therapies in ovarian cancer. Sci Rep 2019;9:18678.

54 Cassidy T, Craig M. Determinants of combination GM-CSF immunotherapy and oncolytic virotherapy success identified through in silico treatment personalization. PLoS Comput Biol 2019;15:e1007495 
Correction: Quantitative systems pharmacology model predictions for efficacy of atezolizumab and nab-paclitaxel in triple-negative breast cancer

Wang H, Ma H, Sové RJ, et al. Quantitative systems pharmacology model predictions for efficacy of atezolizumab and nab-paclitaxel in triple-negative breast cancer. $J$ Immunother Cancer 2021;9:e002100. doi: 10.1136/jitc-2020-002100

This article has been corrected since it first published. The provenance and peer review statement has been added.

Open access This is an open access article distributed in accordance with the Creative Commons Attribution Non Commercial (CC BY-NC 4.0) license, which permits others to distribute, remix, adapt, build upon this work non-commercially, and license their derivative works on different terms, provided the original work is properly cited, appropriate credit is given, any changes made indicated, and the use is non-commercial. See http://creativecommons.org/licenses/by-nc/4.0/.

C Author(s) (or their employer(s)) 2021. Re-use permitted under CC BY-NC. No commercial re-use. See rights and permissions. Published by BMJ.

J Immunother Cancer 2021;9:e002100corr1. doi:10.1136/jitc-2020-002100corr1

A) Check for updates 